\begin{tabular}{|c|l|}
\hline Title & Generalisations of the Hagerup approximation property to arbitrary von Neumann algebras \\
\hline Author(s) & Caspers, Martijn; OKay asu, Rui; Skalski, A dam; Tomatsu, Reiji \\
\hline Citation & $\begin{array}{l}\text { Comptes Rendus Mathematique, 35266), 507-510 } \\
\text { https:/doi.org/40.1016/.crma.2014.04.003 }\end{array}$ \\
\hline Issue Date & 201406 \\
\hline Doc URL & http://hdl.handle.net/2115/56665 \\
\hline Rights & (C) 2014 A cademie des sciences. Published by Elsevier Masson SA S. All rights reserved. \\
\hline Type & article (author version) \\
\hline File Information & CRM_352_507-.pdf \\
\hline
\end{tabular}

Instructions for use 
Haagerup property for arbitrary von Neumann algebras

\title{
Generalisations of the Haagerup approximation property to arbitrary von Neumann algebras
}

\author{
Martijn Caspers ${ }^{\mathrm{a}}$, Rui Okayasu ${ }^{\mathrm{b}}$ Adam Skalski $^{\mathrm{c}, \mathrm{d}}$ Reiji Tomatsu $^{\mathrm{e}}$ \\ ${ }^{a}$ Fachbereich Mathematik und Informatik der Universität Münster \\ Einsteinstrasse 62, 48149 Münster, Germany \\ ${ }^{\mathrm{b}}$ Department of Mathematics Education, Osaka Kyoiku University, Osaka 582-8582, Japan \\ ${ }^{\mathrm{c}}$ Institute of Mathematics of the Polish Academy of Sciences, ul. Sniadeckich 8, 00-956 Warszawa, Poland \\ d Faculty of Mathematics, Informatics and Mechanics, University of Warsaw, ul. Banacha 2, 02-097 Warsaw, Poland \\ ${ }^{\mathrm{e}}$ Department of Mathematics, Hokkaido University, Hokkaido 060-0810, JAPAN \\ Received $* * * * *$; accepted after revision +++++ \\ Presented by —
}

\begin{abstract}
The notion of the Haagerup approximation property, originally introduced for von Neumann algebras equipped with a faithful normal tracial state, is generalized to arbitrary von Neumann algebras. We discuss two equivalent characterisations, one in terms of the standard form and the other in terms of the approximating maps with respect to a fixed faithful normal semifinite weight. Several stability properties, in particular regarding the crossed product construction are established and certain examples are introduced.
\end{abstract}

To cite this article: ******

Résumé

Généralisations de la propriété d'approximation de Haagerup pour les algèbres de von Neumann arbitraires

La notion de la propriété d'approximation de Haagerup, introduite à l'origine pour les algèbres de von Neumann ayants une trace finie, normale, et fidèle, est généralisée pour les algèbres de von Neumann arbitraires. Nous discutons deux caractérisations équivalentes : une du point de vue de la représentation standard et une autre du point de vue des applications linéaires approximatives liées à un poids fidèle, normal, semifini. Quelques propriétés de permanence, en particulier celles concernantes les produits croisés, sont établies et certains exemples sont introduits.

Pour citer cet article : 


\section{Introduction}

The Haagerup property for a locally compact group has its origins in the celebrated paper [9], where U. Haagerup proved that the length function on the free group is conditionally negative definite. This fact, i.e. the existence on a group $G$ of a proper continuous conditionally negative definite function can be viewed as a natural relaxation of amenability of $G$ and has later proved to be a natural and influential notion in geometric group theory, dynamical systems and operator algebras (see [3]). In particular already in 1983 M. Choda showed in [4] that if $G$ is discrete, then it has the Haagerup property if and only if the group von Neumann algebra of $G$ equipped with its natural trace has a certain von Neumann algebraic approximation property, which came to be known as the Haagerup approximation property for a von Neumann algebra with a faithful normal tracial state. The latter property was also motivated by the study of cocycle actions on von Neumann algebras ([5]). Later, still in the framework of finite von Neumann algebras, it was studied in detail by P. Jolissaint in [10], where he proved in particular that in fact it does not depend on the choice of the faithful normal tracial state.

It is fair to say that the interest in extending the notion of the Haagerup property to infinite von Neumann algebras (or even just considering non-tracial states when building $L^{2}$-approximations on finite von Neumann algebras) was rather limited for several years due to the fact that in general there is no hope to characterise the Haagerup property for a non-discrete group via its von Neumann algebra. In the same spirit as the relation between amenability of $G$ and injectivity of $V N(G)$ breaks down for nondiscrete groups. This changed drastically in the last few years, with the advent of a study of the Haagerup property for discrete and locally compact quantum groups. It was initiated by the study of the dual of the quantum free orthogonal and free unitary group by M. Brannan ([1]) and then continued in several directions (see [6] and references therein). The key factor lies in the fact that the natural (Haar) states on the von Neumann algebras of discrete groups need not be tracial, so to characterise the Haagerup property for a discrete quantum group via its von Neumann algebra, one first needs to develop an understanding of the von Neumann algebraic Haagerup approximation property in absence of a tracial state.

The authors of this note approached this question from two directions: MC and AS in [2] studied a direct variant of the original definition, based on the existence of the approximating completely positive maps which behave well with respect to a given faithful normal weight and whose $L^{2}$-GNS implementations are compact, whereas RO and RT in [12] worked in the framework of standard forms, partly motivated by the approach to injectivity due to A. Torpe ([15]). After the original versions of these preprints were circulated, the current authors observed that in fact the two approaches lead to equivalent notions (although this only becomes clear after the respective theories are fully developed). In this note we summarise the main results regarding the Haagerup property for arbitrary von Neumann algebras, referring to [2] and [12] for the proofs of the presented statements.

We assume throughout that the von Neumann algebras we consider have separable preduals and follow the notation of [14].

Email addresses: martijn.caspers@uni-muenster.de (Martijn Caspers), rui@cc.osaka-kyoiku.ac.jp (Rui Okayasu), a.skalski@impan.pl (Adam Skalski), tomatsu@math.sci.hokudai.ac.jp (Reiji Tomatsu).

1. MC was supported by the grant SFB 878 "Groups, geometry and actions".

2. RO was partially supported by JSPS KAKENHI Grant Number 25800065.

3. AS was partially supported by the Iuventus Plus grant IP2012 043872.

4. RT was partially supported by JSPS KAKENHI Grant Number 24740095. 


\section{Main results}

Let $\mathrm{M}$ be a von Neumann algebra and let $\varphi$ be a normal semifinite faithful weight on $\mathrm{M}$. The GNS Hilbert space arising as the completion of the set $\mathfrak{n}_{\varphi}:=\left\{x \in \mathrm{M}: \varphi\left(x^{*} x\right)<\infty\right\}$ with respect to the norm associated to the scalar product $\langle x, y\rangle_{\varphi}:=\varphi\left(y^{*} x\right)$ will be denoted by $L^{2}(\mathrm{M}, \varphi)$, and the GNS embedding map $\mathfrak{n}_{\varphi} \rightarrow L^{2}(\mathrm{M}, \varphi)$ by $\Lambda_{\varphi}$. If $\Phi: \mathrm{M} \rightarrow \mathrm{M}$ is a normal, completely positive map which is $\varphi$-non-increasing, i.e. it satisfies the inequality $\varphi(\Phi(x)) \leq \varphi(x)$ for all $x \in \mathrm{M}_{+}$, then $\Phi$ induces a bounded map on $L^{2}(\mathrm{M}, \varphi)$ via the continuous linear extension of the prescription $\Lambda_{\varphi}\left(\mathfrak{n}_{\varphi}\right) \rightarrow \Lambda_{\varphi}\left(\mathfrak{n}_{\varphi}\right): \Lambda_{\varphi}(x) \mapsto \Lambda_{\varphi}(\Phi(x))$.

Definition 2.1 Let $\mathrm{M}$ be a von Neumann algebra and let $\varphi$ be a normal semifinite faithful weight on $\mathrm{M}$. We say that $\mathrm{M}$ has the Haagerup approximation property with respect to $\varphi$ if there exists a sequence of completely positive, $\varphi$-non-increasing maps $\left(\Phi_{n}\right)_{n=1}^{\infty}$ on $\mathrm{M}$ such that the associated induced maps on the Hilbert space $L^{2}(\mathrm{M}, \varphi)$ are compact and converge to $I_{L^{2}(\mathrm{M}, \varphi)}$ strongly.

In fact, the Haagerup property automatically implies control over the completely positive maps $\Phi_{n}$.

Proposition 2.2 If a von Neumann algebra $\mathrm{M}$ has the Haagerup property with respect to a normal faithful state $\varphi$ then the completely positive maps $\left(\Phi_{n}\right)_{n=1}^{\infty}$ may be chosen such that $\Phi_{n}(1)=1$ and $\varphi \circ \Phi_{n}=\varphi$.

Recall that if $\mathrm{M}$ is a von Neumann algebra, then a quadruple $(\mathrm{M}, \mathrm{H}, J, \mathcal{P})$ is said to be a standard form of $\mathrm{M}$ if $\mathrm{H}$ is a Hilbert space on which $\mathrm{M}$ is faithfully and non-degenerately represented, $J$ is a conjugatelinear isometry of $\mathrm{H}$ with $J^{2}=1$ and $\mathcal{P} \subset \mathrm{H}$ a self-dual closed convex cone, such that $J M J=M^{\prime}, J$ fixes $\mathcal{P}$ pointwise and $x J x J$ fixes $\mathcal{P}$ globally for each $x \in \mathrm{M}$. The existence (and uniqueness up to unitary equivalence) of standard forms was established in [8].

If $(\mathrm{M}, \mathrm{H}, J, \mathcal{P})$ is a standard form of $\mathrm{M}$ and $T$ is a bounded linear operator acting on $\mathrm{H}$ then we say that $T$ is positive if it preserves the cone $\mathcal{P}$. Similarly, using the fact that if $n \in \mathbb{N}$ then the standard form of the von Neumann algebra $M_{n}(\mathrm{M})$ can be realised in the form $\left(M_{n}(\mathrm{M}), M_{n}(\mathrm{H}), J_{n}, \mathcal{P}^{(n)}\right)$, where $\mathcal{P}^{(n)}$ is a certain cone in the Hilbert space $M_{n}(\mathrm{H})$ (identified as the tensor product of $\mathrm{H}$ and $M_{n}$ equipped with the Hilbert-Schmidt norm), we can also say that $T \in B(\mathrm{H})$ is completely positive if for all $n \in \mathbb{N}$ the natural matrix lifting $T^{(n)}: M_{n}(\mathrm{H}) \rightarrow M_{n}(\mathrm{H})$ preserves the cone $\mathcal{P}^{(n)}$ (see [11] and [13]).

Definition 2.3 Let $\mathrm{M}$ be a von Neumann algebra with a standard form $(\mathrm{M}, \mathrm{H}, J, \mathcal{P})$. We say that $\mathrm{M}$ has the standard form Haagerup approximation property if there exists a sequence of contractive completely positive compact operators $\left(T_{n}\right)_{n=1}^{\infty}$ on $\mathrm{H}$ such that $T_{n} \stackrel{n \rightarrow \infty}{\longrightarrow} I_{\mathrm{H}}$ strongly.

Theorem 2.4 Let $\mathrm{M}$ be a von Neumann algebra with separable predual. The following conditions are equivalent:

(i) M has the standard form Haagerup approximation property;

(ii) $\mathrm{M}$ has the Haagerup approximation property with respect to some normal semifinite faithful weight $\varphi$;

(iii) M has the Haagerup approximation property with respect to every normal semifinite faithful weight $\varphi$.

If $\mathrm{M}$ is a finite von Neumann algebra they are also equivalent to the Haagerup approximation property as defined and studied in [4] and [10].

In view of the above theorem and the comments before we will simply say that a von Neumann algebra $\mathrm{M}$ with separable predual has the Haagerup property if it satisfies the equivalent conditions above. We will postpone the discussion of the proof of Theorem 2.4 to the last part of the article. 
Theorem 2.5 The following classes of (possibly infinite) von Neumann algebras have the Haagerup property:

- injective von Neumann algebras, in particular B(H) ([12, Corollary 2.10]);

- von Neumann algebras of discrete quantum groups with the Haagerup property ([6]); in particular the von Neumann algebras of the duals of the quantum free orthogonal and unitary groups ([7]).

The Haagerup property is also stable under several natural von Neumann algebraic constructions, which we list in the next theorem.

Theorem 2.6 The Haagerup property is stable under the following constructions:

- tensor products: $\mathrm{M}_{1} \bar{\otimes} \mathrm{M}_{2}$ has the Haagerup property if and only if both $\mathrm{M}_{1}$ and $\mathrm{M}_{2}$ have the Haagerup property;

- direct sums: $\bigoplus_{n \in \mathbb{N}} \mathrm{M}_{n}$ has the Haagerup property if and only if each $\mathrm{M}_{n}$ has the Haagerup property;

- passing to the commutant: $\mathrm{M}$ has the Haagerup property if and only if $\mathrm{M}^{\prime}$ does;

- passing to a corner: if $p \in \mathrm{M}$ is a projection and $\mathrm{M}$ has the Haagerup property then p $\mathrm{M} p$ has the Haagerup property, moreover if $\left(p_{n}\right)_{n=1}^{\infty}$ is an increasing sequence of projections in $\mathrm{M}$ strongly convergent to $1_{\mathrm{M}}$ and each $p_{n} \mathrm{M} p_{n}$ has the Haagerup property, then $\mathrm{M}$ has the Haagerup property;

- taking conditional expectations: if $\mathrm{M}$ has the Haagerup property and $\mathrm{N}$ is a von Neumann algebra which is the image of a conditional expectation $E: \mathrm{M} \rightarrow \mathrm{N}$ (not necessarily normal!) then $\mathrm{N}$ has the Haagerup property.

Crucially, the Haagerup property behaves well under crossed products, especially by amenable groups.

Theorem 2.7 Let $G$ be a locally compact group and let $\alpha: G \rightarrow \operatorname{Aut}(\mathrm{M})$ be a continuous action of $G$ on the von Neumann algebra $\mathrm{M}$ (this means that $\alpha$ is a homomorphism and the map $G \ni g \mapsto \alpha_{g}(x) \in \mathrm{M}$ is strongly continuous for each $x \in \mathrm{M})$. Then the following hold:

(i) if $\mathrm{M} \rtimes_{\alpha}$ G has the Haagerup property, then $\mathrm{M}$ has the Haagerup property;

(ii) if $G$ is amenable and $\mathrm{M}$ has the Haagerup property, then $\mathrm{M} \rtimes_{\alpha} G$ has the Haagerup property.

The latter result can also be generalised to crossed products by the actions of locally compact amenable quantum groups with amenable duals.

Recall that the core of a von Neumann algebra $M$ is the crossed product of $M$ by the action of the modular automorphism of a normal semifinite faithful weight on $\mathrm{M}$ (it is known to be independent of the choice of such a weight). Theorem 2.7 implies directly the following corollary.

Corollary 2.8 A von Neumann algebra has the Haagerup property if and only if its core does.

Corollary 2.8 is established separately for the Haagerup approximation property with respect to a normal semifinite faithful weight [2] and for the standard form Haagerup approximation property [12] (see Definitions 2.1 and 2.3). This forms the key to the proof of Theorem 2.4. Following [2] the passage to the semifinite core allows one to prove the implication (ii) $\Longrightarrow$ (iii) in Theorem 2.4 . On the other hand, since the core of any von Neumann $\mathrm{M}$ can be decomposed as a tensor product of a finite von Neumann algebra $\mathrm{N}$ and a $\mathrm{I}_{\infty}$-factor, this shows that for both the Definitions 2.1 and 2.3 the Haagerup property of $\mathrm{M}$ is equivalent to the Haagerup property of $\mathrm{N}$ which in turn agrees with the existing notion of the Haagerup approximation property in the case of finite von Neumann algebras. In addition it deserves to be emphasized that the proof of Proposition 2.2 relies on explicit constructions of the approximating maps providing the stability properties of Theorem 2.7 in terms of Definition 2.1. 


\section{References}

[1] M. Brannan, Approximation properties for free orthogonal and free unitary quantum groups, J. Reine Angew. Math. 672 (2012), 223-251.

[2] M. Caspers and A. Skalski, The Haagerup property for arbitrary von Neumann algebras, preprint, arXiv:1312.1491.

[3] P.A. Cherix, M. Cowling, P. Jolissaint, P. Julg and A. Valette, "Groups with the Haagerup property. Gromovs a-Tmenability," Progress in Mathematics, 197, Basel, 2001.

[4] M. Choda, Group factors of the Haagerup type, Proc. Japan Acad. Ser. A Math. Sci. 59 (1983), no. 5, 174-177.

[5] A. Connes and V. Jones, Property T for von Neumann algebras, Bull. London Math. Soc. 17 (1985), 57-62.

[6] M. Daws, P. Fima, A. Skalski and S. White, The Haagerup property for locally compact quantum groups, J. Reine Angew. Math. (Crelle), to appear, arXiv:1303.3261.

[7] K. de Commer, A. Freslon, M. Yamashita, CCAP for the discrete quantum groups $\mathbb{F} O_{F}$, Comm. Math. Phys., to appear, arXiv:1306.6064.

[8] U. Haagerup, The standard form of von Neumann algebras Math. Scand. 37 (1975), no. 2, 271-283.

[9] U. Haagerup, An example of non-nuclear $C^{*}$-algebra which has the metric approximation property, Invent. Math. 50 (1979), no. 3, 279-293.

[10] P. Jolissaint, Haagerup approximation property for finite von Neumann algebras, J. Operator Theory 48 (2002), no. 3, suppl., 549-571.

[11] Y. Miura and J. Tomiyama, On a characterization of the tensor product of self-dual cones associated to the standard von Neumann algebras, Sci. Rep. Niigata Univ. Ser. A No. 20 (1984), 1-11.

[12] R. Okayasu and R. Tomatsu, Haagerup approximation property for arbitrary von Neumann algebras, preprint arXiv: 1312.1033 .

[13] L.M. Schmitt and G. Wittstock, Characterization of matrix-ordered standard forms of $W^{*}$-algebras, Math. Scand. 51 (1982), no. 2, 241-260 (1983)

[14] M. Takesaki, "Theory of operator algebras II," Springer 2000.

[15] A.M. Torpe, A characterisation of semidiscrete von Neumann algebras in terms of matrix ordered Hilbert spaces, preprint, Matematisk Institut, Odense Universitet, 1981. 